\title{
Class Dismissed: First Course of Neuroradiology Successfully Concluded
}

\author{
M. Reiss-Zimmermann • O. Nikoubashman
}

Received: 25 July 2014 / Accepted: 25 July 2014 / Published online: 8 August 2014

(C) Springer-Verlag Berlin Heidelberg 2014

Back at the annual meeting of the German Society of Neuroradiology in 2011, Professor Martin Wiesmann presented the idea of a new educational course, focussing on the society's neuroradiologists in training.

While existing courses only cover special aspects of neuroradiology, this "intensive course of neuroradiology" followed a fundamentally different strategy: during each of the six biannual meetings, the participants, who were selected by their respective head of department, spent 3 days on a one-on-one base learning the whole spectrum of neuroradiology, including interventional techniques in an animal model. This course was not only instructive for the attendees as well as the tutors, but it also helped to improve the communication between the participating hospitals both on a personal and scientific level. For instance, the attendees and tutors vividly exchanged ideas for clinical and scientific practice, which can be quantified by more than 80 articles published in the past 3 years.

Therefore, we strongly believe that this elaborate course strengthens the future of our society. It is an important pillar for the training of future neuroradiologists and for the establishment of strong networks within the neuroradiological society. The convincing concept of the course can also be measured by the fact that a second course is already running and a third one will start in January 2015.

In the name of the attendees of the first course and with great respect for the (sometimes invisible) work we would like to thank Prof. Wiesmann and his team for this intense and very educational course.

M. Reiss-Zimmermann $(\square)$

Department of Neuroradiology, Leipzig University Hospital, Leipzig, Germany

e-mail: martin.reiss-zimmermann@medizin.uni-leipzig.de

O. Nikoubashman

Department of Diagnostic and Interventional Neuroradiology, University of Aachen,

Aachen, Germany 